\section{SAT0615 THE RS11803505 IL-23R POLYMORPHISM IS A RISK FACTOR FOR THE DEVELOPMENT OF MSUS-DETECTED EROSIONS IN PATIENTS WITH SYSTEMIC LUPUS ERYTHEMATOSUS}

\author{
C. Perricone ${ }^{1}$, F. Ceccarelli ${ }^{1}$, V. Iorgoveanu ${ }^{1}$, C. Ciccacci $^{2}$, S. Rufini ${ }^{2}$,
} C. Alessandri ${ }^{1}$, F.R. Spinelli ${ }^{1}$, E. Cipriano ${ }^{1}$, G. Novelli ${ }^{2}$, G. Valesini ${ }^{1}$, P. Borgiani ${ }^{2}$, F. Conti ${ }^{1}$. ${ }^{1}$ Lupus Clinic, Rheumatology, Department of Internal Medicine, Sapienza University of Rome; ${ }^{2}$ Department of Biomedicine and Prevention, University of Rome Tor Vergata, Rome, Italy

Background: Articular involvement is a frequent and invalidating manifestation in patients with Systemic Lupus Erythematosus (SLE) ranging from arthralgia to erosive arthritis. Bone erosions are far less common in SLE but require a different management and may display a distinct pathogenesis. Musculoskeletal ultrasonography (MSUS) has proven useful in the assessment of inflammatory arthritis and it is now known that MSUS is more sensitive than radiography in detecting bone erosions.

Objectives: We aimed at analyzing, in a large monocentric cohort of SLE patients who suffered from arthritis, the relationship between 31 polymorphisms in 18 genes previously associated with SLE or other autoimmune/inflammatory arthritis [1] and the presence of bone erosions detected by MSUS

Methods: Consecutive SLE patients who complained articular involvement (defined by 1997 ACR criteria, i.e. $\geq 2$ joints with tenderness, swelling or effusion) were enrolled. Patients RF+ve and/or anti-CCP+ve were excluded. Thirty-one SNPs in 18 genes (STAT4, IL10, IL23R, IRAK1, PSORS1C1, HCP5, MIR146a, PTPN2, ERAP1, ATG16L1, IRGM, TRAF3IP2, VKORC1, CCR5, ATG5, TRAF6, TNFSF4, CD40) were genotyped by allelic discrimination assays. Bilateral highresolution MSUS of the hands and feet was performed. Erosion number was recorded, the presence of erosive damage was assessed with a dichotomous score.

Results: Clinical and laboratory features of the 95 enrolled patients are described in table.

\begin{tabular}{|c|c|c|}
\hline Clinical and laboratory features & $\mathrm{N}$. & $\%$ \\
\hline Sex & 92 & 96.8 \\
\hline Age $($ mean $\pm S D)$ & \multicolumn{2}{|c|}{$43.38 \pm 11.49$} \\
\hline Age at diagnosis (mean $\pm S D$ ) & \multicolumn{2}{|c|}{$31.14 \pm 11.83$} \\
\hline Anti-dsDNA & 69 & 72.6 \\
\hline MSUS Erosive score (mean \pm SD) & \multicolumn{2}{|c|}{$0.44 \pm 1.34$} \\
\hline MSUS Erosions (yes/no) & $15 / 80$ & 15.8 \\
\hline
\end{tabular}

The presence of MSUS-detected erosions was demonstrated in 15/95 (15.8\%) patients, with a mean $\pm S D$ of $0.44 \pm 1.34$. Bone erosions were more frequently observed at II metacarpophalangeal joint of the hands $(29.4 \%)$, followed by the $\mathrm{V}$ metatarsophalangeal joint of the feet (16.1\%). An association was found between anti-dsDNA and the presence of bone erosions ( $P=0.009$, Fisher's exact test); indeed, all the 15 patients (100\%) with bone erosions were anti-dsDNA positive vs $54 / 80(67.5 \%)$ of those without erosions. After Bonferroni correction, IL-23R rs11803505 was found to be associated with the presence of MSUS-detected bone erosions both at genotypic $\left(P_{c}=0.045\right)$ and at the allelic level $\left[P_{c}=0.022\right.$, $\mathrm{OR}=8.27(95 \% \mathrm{Cl} 1.02-66.90)]$. The association of bone erosions with anti-dsDNA and IL-23R11803505 genotype was confirmed in a logistic regression analysis including also age, sex and disease duration ( $\mathrm{P}=0.005$ and $\mathrm{P}=0.02$, respectively). Conclusions: Our study is the first reporting a contribution of IL-23R gene to MSUS-detected erosive damage in SLE. IL-23R gene is a key player in several inflammatory conditions such as psoriatic arthritis, and variants of this gene have already been associated with peripheral erosive disease [2]. Moreover, we confirmed an association between anti-dsDNA and bone erosions in SLE [3], suggesting that the presence of these antibodies is linked with a more aggressive inflammatory pattern of disease.

\section{References:}

[1] Ciccacci C, Perricone C, et al. PLoS One. 2014.

[2] Jadon D, Tillett W, et al. Rheumatology (Oxford). 2013.

[3] Galvão V, Atta AM, et al. Joint Bone Spine. 2009.

Disclosure of Interest: None declared

DOI: 10.1136/annrheumdis-2017-eular.5989

\section{SAT0616 DIAGNOSTIC MODELING OF SYSTEMIC LUPUS ERYTHEMATOSUS BASED ON NEW AND TRADITIONAL AUTOANTIBODIES- DEMONSTRATION OF FEASIBILITY}

P. Budde ${ }^{1}$, H.-D. Zucht ${ }^{1}$, J. Schulte-Pelkum ${ }^{1}$, D.S. Wirtz ${ }^{1}$, P. Rengers ${ }^{1}$, T. Witte ${ }^{2}$, P. Schulz-Knappe ${ }^{1}$, M. Schneider ${ }^{3}$. ${ }^{1}$ Protagen Ag, Dortmund; ${ }^{2}$ Department of Clinical Immunology, Medical School Hannover, Hannover; ${ }^{3}$ Policlinic of Rheumatology, Heinrich-Heine-University Düsseldorf, Düsseldorf, Germany

Background: Given the heterogeneity of clinical presentations, the diagnosis of Systemic Lupus Erythematosus (SLE) can be challenging, in particular in those patients presenting with early or incomplete disease, or with overlapping or atypical features. Autoantibodies (AABs) are important in aiding the clinical diagnosis of SLE, with some few AABs, anti-double-stranded DNA (dsDNA), anti-Smith (Sm), and anti-ribosomal $P$ (riboP) being highly associated with SLE. As none of the traditional AABs has sufficient sensitivity to achieve diagnosis of
SLE, current testing is based on measuring multiple $A A B$ assays either in parallel or serial. We have recently identified novel AABs in SLE, which hold promise for improving diagnostic testing of SLE (1). We have developed quantitative ELISA-prototypes for five new AABs, which were tested in combination with traditional AABs.

Objectives: The objectives of this study were to evaluate the diagnostic value of novel $A A B s$ and to screen for an optimized combination of novel and traditional AABs using logistic regression to increase the diagnostic accuracy of SLE testing. Methods: Serum samples were obtained from 156 SLE patients with European ancestry at the rheumatology department of the Heinrich-Heine University (Düsseldorf, Germany), and Hannover Medical School (Hannover, Germany). SLE samples were compared against 126 samples from autoimmune diseases (AID; myositis: $n=20$; Sjögren's syndrome $(S j S): n=31$; rheumatoid arthritis (RA) $\mathrm{n}=36$; systemic sclerosis (SSc): $n=39$ ), and 77 healthy control samples. Prototype bead based ELISAs were developed for five novel antigens described in (1). Traditional diagnostic AABs were measured using IVD ELISAs and included: SSA/Ro60, SSA/Ro52, La/SSB, Sm, RNP, dsDNA, Scl70, CENPB, Jo-1, CCP, phospholipid and dsDNA. Optimized marker combinations of new and traditiona markers were tested using logistic regression and receiver operating curve analysis (ROC)

Results: When comparing 156 SLE patients with 203 control samples, the area under the curve (AUC) of the five novel SLE ELISAs ranged from 0.63 to 0.75 . A cut-off was set at a specificity of $95 \%$ and yielded a sensitivity ranging from $13.5 \%$ to $21.2 \%$ for the five novel assays. The sensitivity and specificity of new ELISAs was comparable to traditional ELISAs, which was in this cohort for anti-dsDNA $35 \%$ and $97 \%$, anti-Sm $15 \%$ and $97 \%$, and anti-RiboP $26 \%$ and $97 \%$. A logistic regression model was used to combine the results of multiple tests. Compared to a logistic regression with traditional assays, a logistic regression with novel markers achieved higher sensitivity by pertaining high specificity. The logistic regression model based on a multimarker IVD assay with ten extracted nuclear antigens (ENA) yielded an AUC of 0.87 and a sensitivity of $58 \%$ at a specificity of $95 \%$. By contrast, the optimal combination of traditional and novel ELISAs reached an AUC of 0.92 and a sensitivity of $75 \%$ at a specificity of $95 \%$.

Conclusions: This study demonstrates the feasibility of combining test results of novel and traditional AABs using logistic regression to increase the diagnostic accuracy for SLE. Further studies are required to assess the impact of different ethnicities on marker selection and algorithm performance.

References:

[1] Budde P et al. (2016). Lupus. (8):812-22.

Disclosure of Interest: P. Budde Employee of: Protagen AG, H.-D. Zucht Employee of: Protagen AG, J. Schulte-Pelkum Employee of: Protagen AG, D. Wirtz Employee of: Protagen AG, P. Rengers Employee of: Protagen AG, T. Witte: None declared, P. Schulz-Knappe Employee of: Protagen AG, M. Schneider Consultant for: Protagen AG

DOI: 10.1136/annrheumdis-2017-eular.4995

\section{SAT0617 SALIVARY GLAND ULTRASOUND CAN DIFFERENTIATE PRIMARY SJÖGREN'S SYNDROME FROM SYSTEMIC DISEASES WITH SALIVARY GLAND INVOLVEMENT}

K. Delli ${ }^{1}$, J.F. Van Nimwegen ${ }^{2}$, S. Arends ${ }^{2}$, A.J. Stel ${ }^{2}$, M. Van den Berge ${ }^{3}$, W.F. Bierman ${ }^{4}$, H. Bootsma ${ }^{2}$, F.K.L. Spijkervet ${ }^{1}$, A. Vissink ${ }^{1}$ on behalf of EULAR US-pSS Study Group. ${ }^{1}$ Oral and Maxillofacial Surgery; ${ }^{2}$ Rheumatology \& Clinical Immunology; ${ }^{3}$ Pulmonology; ${ }^{4}$ Internal Medicine, Infectious Diseases Service, University of Groningen, University Medical Center Groningen, Groningen, Netherlands

Background: Ultrasound of the major salivary glands (SGUS) has shown high diagnostic accuracy for primary Sjögren's syndrome (pSS) [1,2]. None of the existing studies, however, included a control group of patients with sarcoidosis, amyloidosis, human immunodeficiency virus (HIV) or hepatitis C (HCV) infection [2]. These diseases are known to frequently affect the major salivary glands, cause dry mouth or may have histopathology similar to pSS.

Objectives: To assess the diagnostic accuracy of SGUS to differentiate pSS from systemic diseases with salivary gland involvement.

Methods: Twenty consecutive patients fulfilling the AECG criteria for pSS and 20 consecutive patients with well-established systemic diseases, i.e., 5 patients with sarcoidosis, 5 patients with amyloidosis, 5 patients with HIV infection and 5 patients with HCV infection, were included. Echogenicity, parenchymal homogeneity, presence of hypoechogenic areas and clearness of posterior glandular border of both parotid and submandibular salivary glands were scored independently by two blinded observers and rated according to the Hocevar et al scoring system (ultrasound total score (UTS), range: 0-48) [3]. The optimal cut-off point was determined by using ROC curve. The agreement between SGUS positivity and classification as pSS was assessed.

Results: The median age of patients with pSS and systemic diseases was 50 and 53 years, respectively. Daily complaints of dry mouth longer than 3 months, need of liquid to swallow food and recurrent or persistent swelling of the major salivary glands were reported by $95 \%, 85 \%$ and $70 \%$ of patients with pSS compared to $45 \%, 30 \%$ and $20 \%$ of patients with systemic diseases. The accuracy of SGUS to discriminate pSS was excellent, with an area under ROC of 0.91 and optimal cut off point of 15. The agreement between SGUS and diagnosis for pSS was good $(\kappa=0.75$, percentage of absolute agreement 87.5$)$ with a sensitivity of $90 \%$, 